\title{
Almacenamiento de carbono en pastos naturales de la subcuenca del Canipaco, Huancayo
}

\author{
Carbon storage in natural pastures of the Canipaco \\ subbasin, Huancayo
}

\author{
Christian Max Quispe Navarro \\ Universidad Continental \\ christmax1@hotmail.com
}

\author{
Fernando Quispe Contreras ${ }^{2}$ \\ Universidad Nacional del Centro \\ del Perú
}

\author{
Raul Marino Yaranga Cano ${ }^{3}$ \\ Universidad Nacional del Centro \\ del Perú
}

\section{RESUMEN}

El objetivo de este estudio fue evaluar la correlación de pesos de biomasa aérea y radicular de las especies Calamagrostis vicunarum, Festuca dolichophylla y Muhlenbergia ligularis y sus capacidades de almacenamiento de carbono. La recolección de datos se realizó en tres zonas representativas tomando en cuenta factores como la fisonomía vegetativa, fisiografía, topografía y exposición hacia los puntos cardinales, denominándolas zonas I, II y III, equivalentes a tres poblaciones diferentes, las que fueron evaluadas aplicando el método de muestreo simple con distribución sistemática. Las unidades muestrales fueron parcelas cuadradas de $4 m^{2}$. La extracción de muestras vegetativas fueron tomadas al azar desde las parcelas, luego lavadas y oreadas a temperatura ambiente bajo techo antes de su traslado a laboratorios para el secado final, pesajes de biomasa y análisis del contenido de carbono. El procesamiento de datos del inventario para la estimación de la Biomasa y correlaciones fue por especie y zonas, luego a partir de los resultados de biomasa y mediante el factor de conversión se calcularon el contenido de carbono almacenado. Entre los resultados podemos mencionar que los promedios de carbono almacenados son: Calamagrostis vicunarum: 0,754 tC/ha; Festuca dolicophylla: 1,638 tC/ha y Muhlenbergia ligularis: $0,743+C / h a$. En conclusión, por la significancia de la correlación, el peso de la biomasa aérea puede permitir la estimación del peso de la biomasa radicular. El promedio de carbono almacenado por las tres especies juntas alcanza a 3,14 tC/ha.

\section{ABSTRACT}

The objective is to evaluate the correlation of aerial and root biomass weights of the Calamagrostis vicunarum, Festuca dolichophylla and Muhlenbergia ligularis species and their carbon storage capacities. The data collection was realized in three representative areas taking into account factors such as vegetative physiognomy, physiography, topography and exposure to cardinal points, called them I, II and III zones, corresponding to three different towns, which were evaluated using the simple sampling with systematic distribution method. The sampling units were square plots of $4 \mathrm{~m}^{2}$. The vegetative samples removal were taken randomly from the plots, then washed and aired at environmental temperature in a closed room before being transferred to laboratories for the final drying, weighed biomass and carbon content analysis. The inventory data processing for the biomass estimating and correlations was by species and areas, then from the biomass results and by the conversion factor were calculated the stored carbon content. As results, we can note that the stored carbon average are: Calamagrostis vicunarum: 0,754 +C/ha; Festuca dolicophylla: 1,638 †C/ha and Muhlenbergia ligularis: 0,743 tC/ha. In conclusion, because of the correlation significance, the air biomass weight may allow root biomass weight estimation. The stored carbon average for these three species together reaches $3,14+\mathrm{C} / \mathrm{ha}$.

Keywords: Carbon, natural pastures, biomass, storage.

Palabras clave: Carbono, pastos naturales, biomasa, almacenamiento

Historial del artículo:

Recibido: 22 de noviembre de 2014. Aprobado: 4 de setiembre de 2015. Disponible en línea: 30 de diciembre de 2015

1 Ingeniero Forestal y Ambiental, investigador de la Universidad Continental.

2 Ingeniero Forestal, docente cesante de la Universidad Nacional del Centro del Perú.

3 Ingeniero Zootecnista, docente de la Universidad Nacional del Centro del Perú. 


\section{INTRODUCCIÓN}

El carbono es el elemento químico fundamental de los compuestos orgánicos, que circula por los océanos, la atmosfera, el suelo y subsuelo. Estos son considerados depósitos de carbono. El carbono pasa de un depósito a otro por medio de procesos químicos, físicos y biológicos. La atmosfera es el menor y el más dinámico de los reservorios del ciclo del carbono. Mientras tanto los cambios que sufren en este reservorio tienen una estrecha relación con los cambios del ciclo global de carbono y del clima.

Gran parte del carbono presente en la atmosfera ocurre en la forma de dióxido de carbono (CO2), en menor proporción el carbono atmosférico se presenta en forma de metano $(\mathrm{CH} 4)$, todos estos son considerados gases de efecto invernadero, que contribuyen con el equilibrio térmico de la tierra. Cualquier actividad relacionada al suelo que modifique la cantidad de biomasa en la vegetación y en el suelo tiene el potencial de alterar la cantidad de carbono almacenado y emitido hacia la atmosfera, lo que influencia directamente en la dinámica del clima de la tierra (1).

En los últimos años una serie de actividades humanas han producido la excesiva emisión de gases de efecto invernadero, principalmente de dióxido de carbono (CO2), que es responsable casi del 50 - $60 \%$ del calentamiento global previsto para los próximos años. De esta manera, se ha sobrepasado la capacidad de absorción de la tierra; con lo que se eleva el calor retenido en la superficie terrestre y aumenta gradualmente la temperatura global del planeta (2). Es decir el mayor aporte humano a los gases de efecto invernadero es $\mathrm{CO} 2$, el cual ha aumentado principalmente desde el inicio de uso masivo de los combustibles fósiles en los países industrializados. Actualmente se consume cien veces más carbón que a fines del siglo XVIII y el petróleo ha visto incrementado su consumo en más de doscientas veces sólo durante el siglo XX. (3). Por lo tanto las concentraciones de $\mathrm{CO} 2$ han aumentado en un $40 \%$ desde la era preindustrial debido, en primer lugar, a las emisiones derivadas de los combustibles fósiles y, en segundo lugar, a las emisiones netas derivadas del cambio de uso del suelo (4).

Según los resultados del Grupo de Trabajo Il del Cuarto Informe Científico AR4 del Panel Intergubernamental de Cambio Climática (IPCC por sus siglas en inglés) el Perú como parte de la región andina tropical, es uno de los países que se verán más afectados por las consecuencias del cambio climático (5). El cambio Climático tendrá impactos en las partes altas de las montañas. Las mediciones de campo en la cordillera central de los Andes indican una tasa de calentamiento que supera el promedio registrado en las partes bajas y las proyecciones futuras estiman que el calentamiento de la superficie en los Andes podría ser el doble de lo que se observará en las tierras bajas de aquí al final del siglo (6).

La Convención Marco de las Naciones Unidas sobre Cambio Climático (CMNUCC) tiene como objetivo estabilizar la concentración de gases de efecto invernadero en la atmosfera y evitar llegar a un nivel de interferencia antropogénica peligrosa. De esta manera el Perú como país en desarrollo ha asumido las responsabilidades descritas en el artículo 4, párrafo 1, de la Convención, en la que señala que se debe elaborar, actualizar y facilitar a la Conferencia de las Partes, inventarios nacionales de las emisiones antropogénicas por las fuentes y de la absorción por los sumideros de todos los gases de efecto invernadero $(7,8)$.

En el marco de la CMNUCC, la primera Conferencia de las Partes decidió elaborar un Protocolo de acciones para implementar compromisos asumidos en la Convención. Este se adoptó en Kioto, Japón, en el año 1997 (2). Este protocolo no sólo contempla las emisiones de gases de efecto invernadero (GEI) a la atmósfera. También considera el papel de los sumideros artificiales, no ha considerado el sumidero natural según sus artículos 3.3 y 3.4 (9).

La modalidad más importante que el Perú aplica a la mitigación es el Mecanismo de Desarrollo Limpio (MDL), uno de los mecanismos del Protocolo de Kyoto que ayuda a cumplir con los objetivos de mitigación. El MDL permite además que países en desarrollo certifiquen sus reducciones de emisiones de GEI y puedan vender dichos certificados a los países industrializados con compromisos de reducción de emisiones. El Perú es parte de la CMNUCC desde 1993 y el protocolo de Kyoto desde el 2002. A través de su Segunda y Tercera Comunicación Nacional, el Perú cumple con su compromiso de informar a las Partes. Y según el inventario Nacional de emisiones de Gases de Efecto Invernadero año 2000, el CO2 es el principal $\mathrm{GEI}$, representando el 73,8 \% del inventario Nacional (7).

El departamento de Junín es una de las regiones del Perú más vulnerables al Cambio Climático (10), con respecto a las proyecciones del clima en la cuenca del Mantaro al 2030 se tendrá un progresivo incremento de las temperaturas máximas y mínimas en toda la cuenca, del orden de $0,9{ }^{\circ} \mathrm{C}$ en promedio respecto al periodo de referencia 1971 - 2000, mientras las proyecciones de la precipitación algunos modelos indican reducciones de hasta $-30 \%$ mientras otros proyectan incrementos de hasta $+15 \%(11,12)$.

Conservar y fomentar los sumideros de carbono es luchar contra el cambio climático. Los sumideros de carbono pueden ser utilizados para el cumplimiento 
de los compromisos de cada estado en la limitación de emisiones de GEI.

El departamento de Junín cuenta con una superficie de más de 1000000 de ha con praderas altoandinas, aproximadamente un cuarto de su superficie total; ecosistemas que retiran carbono de la atmosfera brindando el servicio ambiental de sumideros. Los sumideros almacenan carbono en compuestos orgánicos que conforman la biomasa y la materia orgánica de los suelos y constituyen una de las formas de mitigación del efecto invernadero, sin embargo existe desconocimiento del potencial que tiene este ecosistema para capturar y almacenar Carbono, razón por la cual las poblaciones vienen degradando estos pastizales a través del sobrepastoreo, excediendo su capacidad de carga permitida, no realizan manejo de pastizales, además de practicar la quema de pastizales provocando los incendios forestales y actualmente se viene dando el cambio de uso de tierras, es decir tierras que eran para pastoreo hoy se vienen convirtiendo en tierras con cultivos de maca, contribuyendo así a la emisión de GEl como el CO2.

En el Perú, son escasos los estudios de captura y secuestro de carbono para los ecosistemas de pastizales, desconociéndose el valor potencial de los pastos naturales altoandinos como fijadores de carbono (13). Por esta razón se evaluó la correlación de pesos de biomasa aérea y radicular y el almacenamiento de Carbono fijado por las especies Calamagrostis vicunarum, Festuca dolichophylla y Muhlenbergia ligularis.

La investigación contribuye a sentar una buena base de información técnico-científica incorporando el valor de este ecosistema y proveyendo información útil, que servirá a los tomadores de decisión local, regional y nacional en adoptar políticas sobre medidas de adaptación y mitigación al cambio climático, así también contribuirá con el compromiso del estado peruano como miembro de las partes de la Convención Marco de las Naciones Unidas a proveer información sobre la absorción por los sumideros de carbono en pastizales altoandinos para la formulación de programas nacionales, regionales orientados a mitigar el cambio climático.

\section{MATERIAL Y MÉTODOS}

La subcuenca del Canipaco comprende una extensión de 127 238,69 ha ubicado en el departamento de Junín, en las provincias de Huancayo y Chupaca, abarcando los distritos de Chicche, Colca, Carhuacallanga, Huasicancha, Chacapampa, Chongos Alto y Yanacancha, a una altitud media de 4300 m s.n.m. (figura 1).
Las extensas llanuras de la subcuenca del Canipaco presentan de por sí variadas características en cuanto a su complejidad de amplitud física, topográfica, suelos, humedad, microclimas, cobertura vegetal, donde los pastizales significan ser base y soporte de múltiples servicios desde productivos, económicos y turísticos.

Dada su amplitud territorial se elige tres zonas representativas de estudio, donde con cierta regularidad se visualiza la presencia de la asociación Festucetum Calamagroscetum. Cada zona de muestreo de 10 ha de superficie, con fines de muestreo se constituyen en poblaciones diferentes y cada una son divididas en 25000 parcelas de menor tamaño. Estas zonas tienen como característica lo siguiente: Zona l, se encuentra a una altitud de 4126 m s.n.m., con coordenadas UTM del punto inicial: 460794,8629447; exposición al Sur - Este, pendiente promedio de $15 \%$, suelo arcilloso muy oscuro con profundidad efectiva mayor de $60 \mathrm{~cm}$. Zona II, se ubica a una altitud de 3821 m s.n.m. Punto inicial con coordenadas UTM: 462430,8639337. Exposición al Norte, pendiente promedio de $17 \%$, suelos ligeramente oscuros de mediana profundidad: $40 \mathrm{~cm}$. Zona III, Altitud de 3996 m s.n.m. Punto inicial con coordenadas UTM: 467201,8642679. Exposición al Sur- Oeste. Pendiente de $18 \%$, suelo marrón oscuro de $35 \mathrm{~cm}$, de profundidad las que fueron evaluadas aplicando el método de muestreo simple con distribución sistemática. Las unidades muestrales fueron parcelas cuadradas de $4 \mathrm{~m}^{2}$. Se obtuvo un número de 10 muestras vegetativas por especie desde cada zona, extrayéndolos aleatoriamente a partir de las 25 unidades muestrales ya definidas.

Las muestras se han extraído previa medición de sus alturas además de su diámetro mayor y menor respectivamente. La extracción propiamente dicha fue escarbando con pico el entorno del individuo y desde una profundidad de $30 \mathrm{~cm}$ (caso de Festuca), esta muestra vegetativa se recogía con toda la porción de suelo cuya apariencia era de una torta gigante mientras que en el caso de Calamagrostis y Muhelenbergia, fueron tortas de tamaño medianas extraídas de profundidades menores en razón a la menor longitud de raíz que las caracteriza. Paralelamente se hace el conteo de todos los individuos por especie, en el caso de Festuca, dado su gran tamaño relativo, el conteo fue directo en cada unidad de muestreo y en el caso de las otras especies fue necesario emplear subparcelas de 47,14 cm x 47,14 cm, cuya ubicación estaba en el centro de las parcelas debido a sus menores portes y proporción mayor cuantitativa de individuos por unidad muestral. Luego lavadas y oreadas a temperatura ambiente bajo techo antes de su traslado a laboratorios para el secado final, pesadas de biomasa y análisis del contenido de carbono. El procesamiento de datos del inventario para la estimación de la biomasa y correlaciones fue por especie y por zonas, luego a partir de los resultados de biomasa y mediante 
el factor de conversión se calcularon el contenido de carbono almacenado.

\section{RESULTADOS}

En la tabla 1 se observa que la correlación entre los hojas y diámetro, promedio tanto en poblaciones I y III, además de que el peso de la biomasa aérea y la altura de hojas en zona I también muestra ser significativo a nivel de $\mathrm{P} \leq 0,05$; en caso de Muhlenbergia ligularis, se observa una correlación significativa entre el peso de la biomasa aérea con altura de la planta y promedio de diámetro en población II y altamente significativa

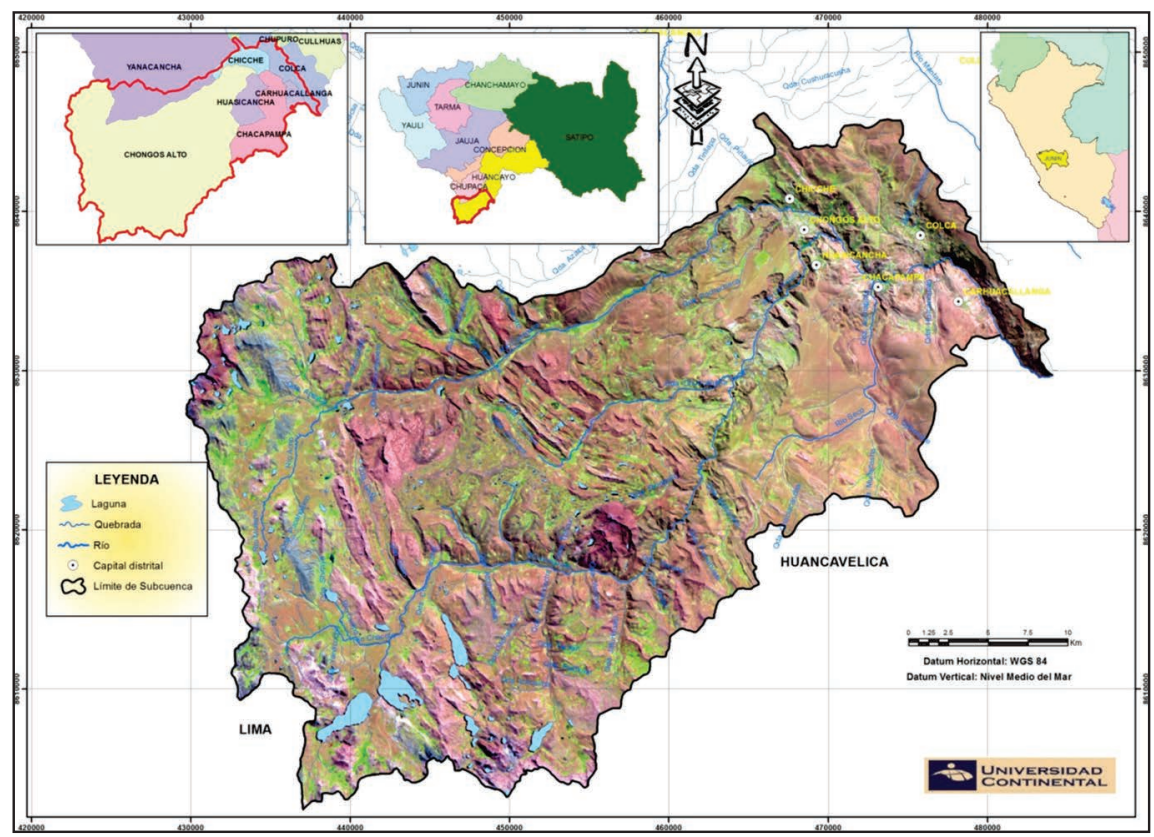

Figura $\mathrm{N}^{\circ}$ 1: Ubicación de la subcuenca del Canipaco.

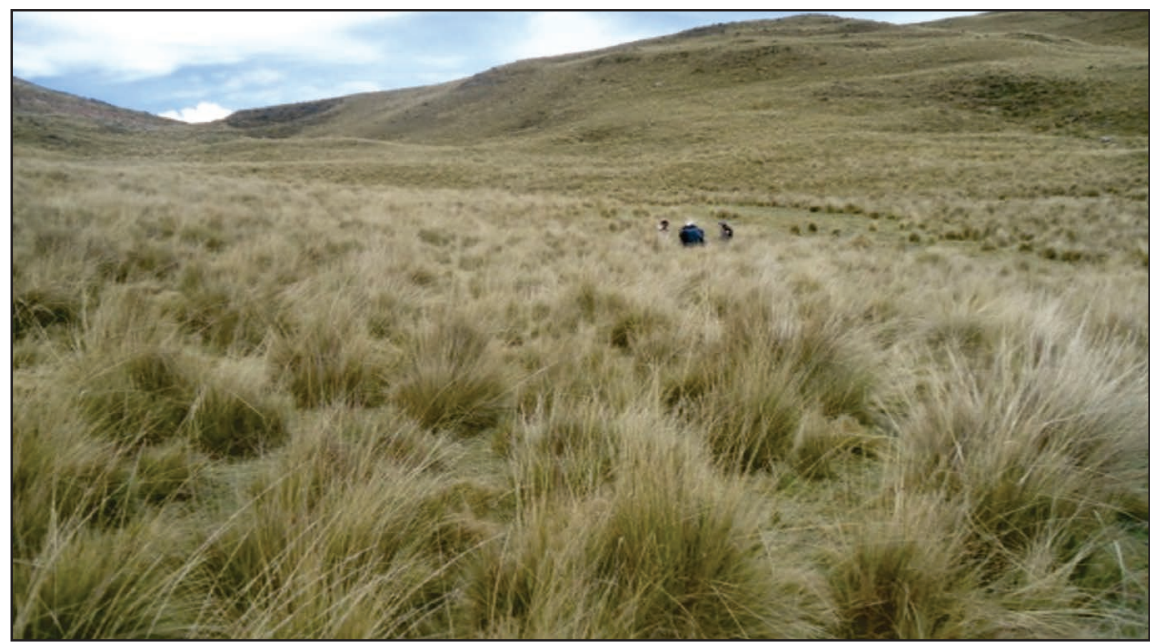

Figura $\mathrm{N}^{\circ}$ 2: Pastizales naturales de la subcuenca del Canipaco.

ítems analizados son bajas. En caso de Calamagrostis vicunarum solo es significativa a nivel de $\mathrm{P} \leq 0,05$ cuando se trata entre la altura promedio de hojas y el promedio de diámetro en poblaciones I y II; mientras tanto en el caso de Festuca dolichophylla, es más notoria la correlación entre la altura total de la planta y promedio de diámetro, entre la altura de entre el peso de biomasa aérea y diámetro promedio en población III.

En la tabla 2 se observa la correlación entre los pesos de biomasa aérea y radicular de las tres especies: Calamagrostis vicunarum, Festuca dolichophylla, y Muhlenbergia ligularis, tanto a nivel general como 
Tabla $N^{\circ}$ 1: Correlación de peso y medidas Agronómicas de la biomasa aérea.

\begin{tabular}{|c|c|c|c|c|}
\hline & Correlación & $\begin{array}{c}\text { Calamagrostis } \\
\text { vicunarum }\end{array}$ & $\begin{array}{c}\text { Festuca } \\
\text { dolichophylla }\end{array}$ & $\begin{array}{c}\text { Muhlenbergia } \\
\text { ligularis }\end{array}$ \\
\hline \multirow[t]{4}{*}{ Zona I } & $\begin{array}{l}\text { Altura total de planta y } \\
\text { promedio de diámetro }\end{array}$ & 0,5520 & $0,7539^{*}$ & \\
\hline & $\begin{array}{l}\text { Altura promedio de hojas } \\
\text { y promedio de diámetro }\end{array}$ & $0,6936^{*}$ & $0,7143^{*}$ & \\
\hline & $\begin{array}{l}\text { Peso de Biomasa aérea y } \\
\text { altura de hojas }\end{array}$ & 0,5845 & $0,7026^{*}$ & \\
\hline & $\begin{array}{l}\text { Peso de Biomasa aérea y } \\
\text { diámetro promedio }\end{array}$ & 0,6030 & 0,5888 & \\
\hline \multirow[t]{3}{*}{ Zona II } & $\begin{array}{l}\text { Altura total de planta y } \\
\text { promedio de diámetro }\end{array}$ & $0,9222^{* *}$ & 0,3626 & 0,6757 \\
\hline & $\begin{array}{l}\text { Peso de Biomasa aérea y } \\
\text { altura de hojas }\end{array}$ & $0,9019^{* *}$ & $-0,0023$ & $0,8554^{*}$ \\
\hline & $\begin{array}{l}\text { Peso de Biomasa aérea y } \\
\text { diámetro promedio }\end{array}$ & $0,9011^{* *}$ & 0,2322 & $0,8800^{*}$ \\
\hline \multirow[t]{4}{*}{ Zona III } & $\begin{array}{l}\text { Altura total de planta y } \\
\text { promedio de diámetro }\end{array}$ & $-0,2554$ & $0,6956^{*}$ & $-0,2324$ \\
\hline & $\begin{array}{l}\text { Altura promedio de hojas } \\
\text { y promedio de diámetro }\end{array}$ & $-0,3364$ & $0,6946^{*}$ & 0,0606 \\
\hline & $\begin{array}{l}\text { Peso de Biomasa aérea y } \\
\text { altura de hojas }\end{array}$ & 0,4850 & 0,5543 & $-0,0067$ \\
\hline & $\begin{array}{l}\text { Peso de Biomasa aérea y } \\
\text { diámetro promedio }\end{array}$ & $-0,0840$ & 0,4460 & $0,8087^{* *}$ \\
\hline
\end{tabular}

* Significativo ${ }^{* *}$ Altamente Significativo

por cada zona de espacio estudiado. Se observa que en las especies de porte bajo como son las Calamagrostis vicunarum y Muhlenbergia ligularis, la correlación es significativa nivel de $\mathrm{P} \leq 0,05$ y $\mathrm{P} \leq 0,01$ respectivamente, lo que significa que a un cambio en la raíz de $1 \mathrm{~g}$, la variación del peso de la biomasa aérea de Calamagrostis vicunarum ocurre en 1,74 g, mientras tanto en Muhlenbergia ligularis el cambio de $1 \mathrm{~g}$ del peso de la raíz, la biomasa aérea cambia en
1,67 g. La correlación entre pesos de biomasa en la Festuca dolichophylla no es significativo.

Por otro lado en la figura 2 se observan que el promedio de peso de la biomasa aérea con respecto al peso de la biomasa radicular, no son coincidentes, las especies de crecimiento bajo muestran proporciones entre 60 y $70 \%$ de biomasa aérea, mientras que en la Festuca dolichophylla bordea el $80 \%$.

Tabla $N^{\circ}$ 2: Correlación entre biomasa aérea y radicular de especies .

\begin{tabular}{lccc}
\hline $\begin{array}{c}\text { Correlacion/ } \\
\text { Regresion }\end{array}$ & $\begin{array}{c}\text { Calamagrostis } \\
\text { vicunarum }\end{array}$ & $\begin{array}{c}\text { Festuca } \\
\text { dolichophylla }\end{array}$ & $\begin{array}{c}\text { Muhlenbergia } \\
\text { ligularis }\end{array}$ \\
\hline Correlación general & $0,7718^{*}$ & 0,3273 & $0,9066^{* *}$ \\
Correlación zona I & 0,3530 & 0,5178 & $0,9885^{* *}$ \\
Correlación zona II & $0,9494^{* *}$ & $0,7513^{*}$ & $0,8494^{*}$ \\
Correlación zona III & $0,9301^{* *}$ & 0,5146 & 0,2920 \\
Regresión general & 1,7409 & & 1,6738 \\
Regresión zona I & & & 1,3643 \\
Regresión zona II & 2,7527 & 0,7513 & 1,7884 \\
Regresión zona III & 1,4846 & & \\
\hline
\end{tabular}

* Significativo ** Altamente Significativo 


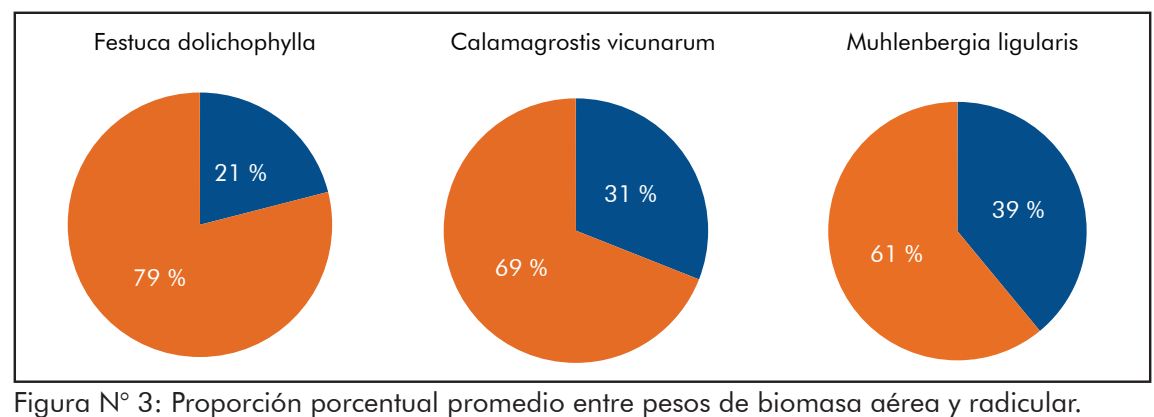

Las muestras vegetativas extraídas para determinar el peso promedio de Biomasa por planta o individuo de las especies estudiadas, fueron tomadas al azar desde las parcelas de muestreo, en dichas muestras se encuentran representados casi todos los tamaños de la población de individuos. La variabilidad de pesos de estas biomasas individuales son notorias en las tres zonas o poblaciones y están expresadas

Tabla № 3: Muestras de Biomasa seca por planta (individuo) según especies (gr).

\begin{tabular}{|c|c|c|}
\hline $\begin{array}{l}\text { Calamagrostis } \\
\text { vicunarum }\end{array}$ & $\begin{array}{c}\text { Festuca } \\
\text { dolichophylla }\end{array}$ & $\begin{array}{c}\text { Muhlenbergia } \\
\text { ligularis }\end{array}$ \\
\hline 20,35 & 128,07 & 9,12 \\
\hline 15,3 & 13,6 & 3,82 \\
\hline 16,3 & 20,36 & 2,19 \\
\hline 10,32 & 85,79 & 7,77 \\
\hline 12,85 & 94,78 & 3,98 \\
\hline 10,07 & 98,44 & 2,77 \\
\hline 5,68 & 169,43 & 2,08 \\
\hline 12,66 & 153,21 & 3,93 \\
\hline 9,2 & 74,36 & 6,92 \\
\hline 7,86 & 266,67 & 20,3 \\
\hline 2,91 & 120,79 & 5,91 \\
\hline 4,12 & 121,55 & 9,65 \\
\hline 5,84 & 171,48 & 5,87 \\
\hline 5,6 & 133,91 & 9,42 \\
\hline 9,49 & 237,9 & 9,4 \\
\hline 14,89 & 147,63 & 18,16 \\
\hline 5,92 & 52,52 & 11,8 \\
\hline 29,12 & 171,36 & 25,3 \\
\hline 5,01 & 242,75 & 11,91 \\
\hline 5,01 & 108,21 & 11,96 \\
\hline 2,7 & 204,03 & 1,89 \\
\hline 3,39 & 84,16 & 2,6 \\
\hline 3,48 & 74,67 & 2,13 \\
\hline 2,01 & 157,65 & 2,13 \\
\hline 14,13 & 103,74 & 4,11 \\
\hline 6,2 & 168,43 & 2,95 \\
\hline 3,92 & 84,54 & 2,1 \\
\hline 4,1 & 137,63 & 3,97 \\
\hline 4,41 & 27,68 & 5,67 \\
\hline 5,06 & 26 & 1,45 \\
\hline
\end{tabular}

por la magnitud del valor o nivel de precisión (tablas 3 y 4), lo que denota que las matas o plantas fueron de diferentes tamaños, siendo mayor aun en Calamagrostis vicunarum así como en Muhlenbergia ligularis, y relativamente menor variabilidad entre las muestras de Festuca dolichophylla.

En la zona I (tabla 5), se observa que el promedio de la cantidad de biomasa por parcela más alta corresponde a la especie Festuca dolichophylla, llega a $2,057 \mathrm{~kg} /$ parcela y en segundo lugar ocupa la especie Calamagrostis vicunarum con 0,965 kg/parcela y en tercer lugar Muhlenbergia ligularis alcanza un 0,867 $\mathrm{kg} /$ parcela, todos ellos con una confianza del $95 \%$. El promedio de la última especie es más precisa en relación a las otras, lo que significa que existe una presencia relativamente más homogénea dentro de la zona l.

En la zona II (tabla 6), la cantidad de biomasa acumulada es más baja en las tres especies, sin embargo lo resaltante es que siempre ocupa el primer lugar Festuca dolichophylla con 0,7169 kg/parcela y además presenta un menor error de muestreo es decir un promedio con una precisión ligeramente mayor frente a las otras especies.

En la tercera zona (tabla 7), se observa que los contenidos de biomasa de Calamagrostis vicunarum y Muhlenbergia ligularis bajan aún más en su contenido de biomasa, mientras que Festuca dolichophylla alcanza un valor de $1,006 \mathrm{~kg} /$ parcela con una precisión de la media relativamente más alta que las demás especies. Significa entonces que esta especie está distribuida en la población III con más regularidad que las otras dos especies.

Al observar los pesos por $\dagger$ de la especie Festuca dolichophylla en las zonas I, II y III (tablas 8,9 y 10), se ve un comportamiento relevante y alcanza hasta 5,1425 t/ha, 1,7922 t/ha y 2,5155 t/ha. Siendo la especie la mayor aportante de biomasa en las tres zonas.

En estas mismas tablas 8, 9 y 10 al establecer los límites de confianza respectivos en la estimación del peso de la biomasa y en concordancia a los 
Tabla N 4: Análisis estadístico de Biomasa/planta* según especies.

\begin{tabular}{|c|c|c|c|}
\hline Simbología & $\begin{array}{c}\text { Calamagrostis } \\
\text { vicunarum }\end{array}$ & $\begin{array}{c}\text { Festuca } \\
\text { dolichophylla }\end{array}$ & $\begin{array}{c}\text { Muhlenbergia } \\
\text { ligularis }\end{array}$ \\
\hline$x^{-}$ & 0,0085960 & 0,122711333 & 0,007042000 \\
\hline$s^{2}$ & 0,0000375 & 0,004267919 & 0,000034880 \\
\hline $\mathrm{s}$ & 0,0061287 & 0,065329311 & 0,005905929 \\
\hline$\pm \mathrm{e}$ & $\pm 0,0022883$ & $\pm 0,024392631$ & $\pm 0,002205171$ \\
\hline e \% & 26,5500000 & 19,878059010 & 22,050000000 \\
\hline
\end{tabular}

*Biomasa/unidad vegetativa o planta en $\mathrm{kg}$.

Tabla $N^{\circ}$ 5: Biomasa por especies a nivel muestra de la zona I.

\begin{tabular}{lcccc}
\hline Especie & $\mathrm{X}^{-}$ & $\mathrm{s}^{2}$ & $\mathrm{~s}$ & $\pm \mathrm{e}$ \\
\hline Calamagrostis & 0,96550000 & 0,319768670 & 0,565480920 & 0,233430520 \\
Festuca & 2,05700000 & 0,898434500 & 0,947857850 & 0,391275740 \\
Muhlenbergia & 0,86701104 & 0,146853144 & 0,383214227 & 0,158190833 \\
\hline
\end{tabular}

Tabla $N^{\circ}$ 6: Biomasa por especies a nivel muestra de la Zona II.

\begin{tabular}{lcccc}
\hline Especie & $\mathrm{X}^{-}$ & $\mathrm{s}^{2}$ & $\mathrm{~s}$ & $\pm \mathrm{e}$ \\
\hline Calamagrostis & 0,47656224 & 0,089618220 & 0,29930000 & 0,12350000 \\
Festuca & 0,71698264 & 0,145301006 & 0,38118369 & 0,15735000 \\
Muhlenbergia & 0,62870976 & 0,137212890 & 0,38118369 & 0,15291044 \\
\hline
\end{tabular}

Tabla $N^{\circ}$ 7: Biomasa por especies a nivel muestra de la Zona III.

\begin{tabular}{lcccc}
\hline Especie & $\mathrm{X}^{-}$ & $\mathrm{s}^{2}$ & $\mathrm{~s}$ & $\pm \mathrm{e}$ \\
\hline Calamagrostis & 0,34659072 & 0,023003074 & 0,151667645 & 0,062600000 \\
Festuca & 1,00622200 & 0,160615937 & 0,400769182 & 0,165440000 \\
Muhlenbergia & 0,17914848 & 0,009120715 & 0,095502433 & 0,039582433 \\
\hline
\end{tabular}

Tabla $N^{\circ}$ 8: Biomasa promedio por especies a nivel de población y ha Zona I.

\begin{tabular}{|c|c|c|c|c|c|}
\hline Especie & $x^{-}$ & $N$ & B. $\mathrm{kg}$ & B. $t / z$ & B.t/ha \\
\hline Calamagrostis & 0,965 & 25000 & 24125 & 24,125 & 2,4125 \\
\hline Festuca & 2,057 & 25000 & 51425 & 51,425 & 5,1425 \\
\hline Muhlenbergia & 0,867 & 25000 & 21675 & 21,675 & 2,1675 \\
\hline Total & 3,889 & -.--_- & 97225 & 97,225 & 9,7225 \\
\hline
\end{tabular}

Tabla $N^{\circ}$ 9: Biomasa promedio por especies a nivel de población y ha Zona II.

\begin{tabular}{|c|c|c|c|c|c|}
\hline Especie & $x^{-}$ & $N$ & B. $\mathrm{Kg} / \mathrm{z}$ & B. $t / z$ & B.t/ha \\
\hline Calamagrostis & 0,4765 & 25000 & 11912,5 & 11,9125 & 1,1912 \\
\hline Festuca & 0,7169 & 25000 & 17922,5 & 17,9225 & 1,7922 \\
\hline Muhlenbergia & 0,6287 & 25000 & 15717,5 & 15,7175 & 1,5717 \\
\hline Total & 1,8221 & ---o-- & 45552,5 & 45,5525 & 4,5551 \\
\hline
\end{tabular}


descriptores estadísticos (tablas 5, 6 y 7) existe con una confiabilidad de $95 \%$, en zona I a nivel de población: Calamagrostis vicunarum: $2,4125 \pm 0,5825 \mathrm{t} / \mathrm{ha}$; Festuca dolichophylla: $5,1425 \pm 0,9577 \mathrm{t} / \mathrm{ha}$ y en Muhlenbergia ligularis: 2,1675 $\pm 0,395 \mathrm{t} / \mathrm{ha}, \mathrm{En}$ zona II, Calamagrostis vicunarum: 1,1912 \pm 0,307
En la tabla 11, se muestran los valores de carbono almacenado por especies en la Zona I. Al fijarse en los contenidos de carbono para el promedio de las biomasas según la parte vegetativa aérea o radicular que se trate. La especie Calamagrostis vicunarum almacena una cantidad de 1,256 t, de carbono/ha,

Tabla № 10: Biomasa promedio por especies a nivel de población y ha Zona III.

\begin{tabular}{lccccc}
\hline Especie & $\mathrm{X}^{-}$ & $\mathrm{N}$ & $\mathrm{B} . \mathrm{Kg} / \mathbf{z}$ & $\mathrm{B} . \mathrm{t} / \mathbf{z}$ & B.t/ha \\
\hline Calamagrostis & 0,3465 & 25000 & 8662,5 & 8,6625 & 0,8662 \\
Festuca & 1,0062 & 25000 & 25155 & 25,155 & 2,5155 \\
Muhlenbergia & 0,1791 & 25000 & 4477,5 & 4,4775 & 0,4477 \\
Total & 1,5318 & $-\cdots--\cdot---$ & 38295 & 38,295 & 3,8294 \\
\hline
\end{tabular}

Tabla № 11: Promedios de biomasa y carbono según partes vegetativas, Zona I.

\begin{tabular}{lccccc}
\hline Especie & Parte vegetativa & Biomasa* & B.t/ha & Carbono** & C.t/ha \\
\hline Calamagrostis & A & 0,6876 & 1,658835 & 0,5197 & 0,862 \\
& R & 0,3124 & 0,753665 & 0,5234 & 0,394 \\
& Tot. & 1,0000 & 2,412500 & $\ldots \ldots$ & 1,256 \\
Festuca & & & & 0,5325 & 2,151 \\
& A & 0,7858 & 4,0409765 & 0,549 \\
Muhlenbergia & R & 0,2142 & 1,1015235 & 0,4992 & 2,700 \\
& Tot. & 1,0000 & 5,1425000 & $\ldots$. & 0,713 \\
& A & 0,6087 & 1,31935725 & 0,5406 & 0,444 \\
& R & 0,3913 & 0,84814275 & 0,5239 & $\ldots$ \\
\hline
\end{tabular}

*Fracción de Biomasa Aérea y radicular.

**factor de conversión de carbono

Tabla № 12: Promedios de biomasa y carbono según partes vegetativas, Zona II.

\begin{tabular}{lccccc}
\hline Especie & Parte vegetativa & Biomasa* $^{*}$ & B.t/ha & Carbono** & C.t/ha \\
\hline Calamagrostis & A & 0,6876 & 0,81906912 & 0,5023 & 0,411 \\
& R & 0,3124 & 0,37213088 & 0,4450 & 0,165 \\
Tot. & 1,0000 & 1,19120000 & $\ldots \ldots$ & 0,576 \\
Festuca & & & & 0,5196 & 0,731 \\
& A & 0,7858 & 1,40831076 & 0,165 \\
Muhlenbergia & R & 0,2142 & 0,38388924 & 0,4320 & 0,896 \\
& Tot. & 1,0000 & 1,79220000 & $\ldots \ldots \ldots$ & 0,503 \\
& A & 0,6087 & 0,95669379 & 0,5264 & 0,334 \\
& R & 0,3913 & 0,61500621 & 0,5432 & 0,837 \\
\hline
\end{tabular}

*Fracción de biomasa aérea y radicular.

**factor de conversión de carbono

t/ha, Festuca dolichophylla: 1,7922 \pm 0,392t/ha; Muhlenbergia ligularis: $1,5717 \pm 0,380 \mathrm{t} / \mathrm{ha}$ y en la zona III, el resultado es como sigue: Calamagrostis vicunarum $0,8662 \pm 0,1565$ t/ha Festuca dolichophylla: $2,5155 \pm 0,4135 \mathrm{t} /$ ha y Muhlenbergia ligularis: $0,4477 \pm 0.0989$ t/ha. de los cuales el 0,862 t, se encuentra en el tallo y/o follaje llamada en este caso parte Aérea y $0,394 \mathrm{t} / \mathrm{ha}$ de carbono se encuentra almacenado en las raíces. La especie Festuca dolichophylla almacena 2,7 t, siendo 2,151 t en aéreo y 0,549 † en la raíz; la especie Muhlenbergia ligularis almacena 1,157 t/ha de los 
cuales 0,713 † es aéreo y 0,444 † es en raíz.

En la tabla 12, se muestran los valores de carbono almacenado por especies en la Zona II. La especie Calamagrostis vicunarum almacena una cantidad de 0,576 t, de carbono/ha, de los cuales el 0,411 t de carbono/ha, se encuentra en el tallo y/o follaje llamada en este caso parte aérea y 0,165 † de carbono/ ha se encuentra almacenado en las raíces. La especie Festuca dolichophylla almacena 0,896 t, siendo 0,731 t en aéreo y 0,165 t en la raíz; la especie Muhlenbergia ligularis almacena $0,837 \mathrm{t} / \mathrm{ha}$ de los cuales $0,503 \mathrm{t}$ es aéreo y 0,334 t es en raíz.

En la tabla 13, se muestran los valores de carbono almacenado por especies en la Zona III. La especie Calamagrostis vicunarum almacena una cantidad de 0,430 t, de carbono/ha, de los cuales el 0,292 t de carbono/ha, se encuentra en el tallo y/o follaje llamada en este caso parte aérea y 0,138 t de carbono/ ligularis la significancia se mantiene en poblaciones I y II; igualmente se observa una significancia de $\mathrm{P} \leq 0,05$ en Festuca dolichophylla en población II. Estos resultados muestran el comportamiento de las especies según factores ambientales que diferencian a las tres poblaciones estudiadas (suelos, orientación del plano con respecto a los puntos cardinales, humedad del suelo). La alta correlación que existe entre los datos de la biomasa aérea y la biomasa radicular, en las especies Calamagrostis vicunarum y Muhlenbergia ligularis, otorgan una gran confiabilidad para asumir el peso de la raíz de manera indirecta con sólo saber el peso total de la biomasa aérea, los cuales amplían los resultados de Ramírez et (14) quienes encontraron coeficiente de correlación entre 0,78 y 0,83, al estudiar Brachiaria humidícola. El porcentaje del peso de la raíz calculado con respecto al peso de la biomasa osciló entre $21 \%$ y $39 \%$, sugieren inferir que no se puede atribuir un porcentaje único cuando se trata de pastos naturales altoandinos. Estos resultados, sugieren que

Tabla $N^{\circ}$ 13: Promedios de biomasa y carbono según partes vegetativas, Zona III.

\begin{tabular}{|c|c|c|c|c|c|}
\hline Especie & Parte vegetativa & Biomasa* & B.t/ha & Carbono** & C.t/ha \\
\hline \multirow[t]{3}{*}{ Calamagrostis } & $A$ & 0,6876 & 0,59559912 & 0,4915 & 0,292 \\
\hline & $\mathrm{R}$ & 0,3124 & 0,27060088 & 0,5101 & 0,138 \\
\hline & Total & 1,0000 & 0,8662 & $\ldots \ldots \ldots$ & 0,430 \\
\hline \multirow[t]{3}{*}{ Festuca } & $A$ & 0,7858 & 1,9766799 & 0,5507 & 1,088 \\
\hline & $\mathrm{R}$ & 0,2142 & 0,5388201 & 0,4205 & 0,226 \\
\hline & Total & 1,0000 & 2,5155 & .......... & 1,314 \\
\hline \multirow[t]{3}{*}{ Muhlenbergia } & $A$ & 0,6087 & 0,27251499 & 0,5526 & 0,150 \\
\hline & $\mathrm{R}$ & 0,3913 & 0,17518501 & 0,4923 & 0,086 \\
\hline & Total & 1,0000 & 0,4477 & ........ & 0,236 \\
\hline
\end{tabular}

*Fracción de biomasa aérea y radicular.

**factor de conversión de carbono

ha se encuentra almacenado en las raíces. La especie Festuca dolichophylla almacena 1,314 t, siendo 1,088 t en aéreo y 0,226 t en la raíz; la especie Muhlenbergia ligularis almacena $0,236 \mathrm{t} / \mathrm{ha}$ de los cuales 0,150 t es aéreo y 0,086 † es en raíz. El promedio de carbono acumulado por las tres especies juntas que resultan del estudio realizado alcanza a 3,13 t/ha.

\section{DISCUSIÓN}

En la correlación entre el peso de la biomasa aérea y algunas medidas agronómicas existe cierta correlación positiva y significativa en la Festuca dolichophylla, mientras que en las otras tiende a ser negativa. Estas correlaciones entre los pesos de biomasa aérea y radicular observadas en las tres poblaciones de espacio evaluado no se mantienen, en Calamagrostis vicunarum son significativos en poblaciones II y III, mientras que en Muhlenbergia es necesario estudiar la diversidad de pastos naturales a fin de determinar las especies más abundantes para establecer los factores de corrección que permitan realizar cálculos indirectos de peso de la biomasa radicular.

Al observar los pesos por t de la especie Calamagrostis vicunarum en las zonas I, II y III (tablas 8,9 y 10) se nota que en áreas con exposición Sur - Este, alcanza los más altos niveles y en aquellas áreas con orientación hacia el Sur - Oeste son más bajos, luego en aquellas áreas con orientación al Norte, ocupa un valor intermedio. La especie Festuca dolichophylla, tiene un comportamiento relevante y alcanza a 5, $1425 \mathrm{t} /$ ha en áreas con exposición al Sur - Este, mientras que con exposición al Sur - Oeste llega a 2,5155 t/ha, llegando a un valor intermedio en las áreas con exposición al Norte. En cuanto a Muhlenbergia ligularis, ésta llega a buenos niveles en áreas con exposición al Sur - Este y Norte, pero baja sensiblemente en aquellas áreas con 
exposición al Sur- Oeste. En general la especie Festuca dolichophylla, es la mayor aportante de biomasa en las tres zonas, esto significa que la especie prospera y se mantiene con regularidad bajo cualquier exposición topográfica y sus diferencias de rendimiento en biomasa que figuran en las diferentes zonas son inherentes básicamente a la gradiente de fertilidad del factor suelo de cada lugar.

En la zona I se observa que el almacenamiento de carbono por ha es mayor para las tres especies en estudio respecto a las Zonas I y II. En la zona II se ve que el almacenamiento de carbono por ha es menor para las tres especies en estudio respecto a la zona I, esto puede explicarse que es debido a la exposición Norte que la caracteriza al área de muestreo y por cierto en razón a la menor profundidad efectiva del suelo que influye en la producción de biomasa. En la zona III los valores promedio de almacenamiento de carbono son aún más pequeños para las especies de Calamagrostis vicunarum y Muhlenbergia ligularis, lo cual puede obedecer a varios factores como: el aspecto edáfico que es más superficial en comparación con las otras áreas y también por la relevante exposición topográfica hacia el Sur-Oeste de la zona en referencia; sin embargo sobresale la especie Festuca dolichophylla con 1,314 t/ha. Reafirmando que ésta especie tiene más regularidad en su presencia y distribución espacial tanto en gradientes diferentes de suelos como en relación a la exposición topográfica variada de las áreas. El promedio de carbono acumulado por las tres especies alcanza a 3,13 t/ha. Equivalente a 11,49 t/ ha de $\mathrm{CO} 2$, si consideramos que una $\dagger$ de carbono equivale a 3,67 † de $\mathrm{CO} 2$, obtenido en función de los pesos moleculares del carbono y del CO2, de 12 / 44 (1). Basado en este resultado puede afirmarse la existencia probable de 296 180,85 + de carbono, equivalentes a 1086983,72 + de CO2 almacenado en las 94 626,47 ha, de pastizales en el Canipaco.

Yaranga $R$, et al (13) investigaron sobre el almacenamiento de carbono en pastos naturales en el distrito de Huasicancha y Chicche en Huancayo y determinaron que la concentración de carbono en las cinco especies de pastos fue superior a 30,0 \%. Así mismo Medrano R, et al (15) investigó sobre el almacenamiento de Carbono en especies predominantes de flora en el lago Chinchaycocha, concluyendo que los totorales y juncos brindan el mayor almacenamiento de carbono con 39,35 tC/ha, luego los pajonales con $15,43+C /$ ha y en menor proporción los bofedales con 6,46 tC/ha; el almacenamiento de carbono en suelos se determinó en 774,76 tC/ha en pajonales y $684,58 \mathrm{tC} / \mathrm{ha}$ en bofedales. Asimismo el INIA investigó sobre determinación de reservas totales de Carbono en diferentes sistemas de uso en el Valle del Mantaro, y el resultado de Carbono total almacenado en el uso de tierra pastizal en la Subcuenca del Shullcas fue de 116,439 t/ha (16).

\section{REFERENCIAS BIBLIOGRÁFICAS}

1. Instituto Nacional de Innovación Agraria. Manual para Evaluación de Carbono en Pequeñas Propiedades Rurales. Huancayo. 201 1. 2,3

2. Ministerio del Ambiente. Guía para la Elaboración de Estrategias Regionales frente al Cambio Climático. 2011. 17,101

3. CONAM. Estrategia Nacional de Cambio Climático. Comisión Nacional de Cambio Climático. 2002.46

4. Grupo Intergubernamental de Expertos sobre el Cambio Climatico. Cambio Climatico 2013. Bases físicas.IPCC 2013.34

5. IPCC, 2007b. Climate Change 2007: Impact and vulnerability, Summary for Policy Maker, Working Group 2 IPCC, Geneva, 2007

6. Stockholm Environment Institute. Evaluación de los impactos del cambio climático en la hidrología de montaña. 2012

7. Ministerio del Ambiente. Segunda comunicación Nacional del Perú a la Convención Marco de las Naciones Unidas sobre Cambio Climático. 2010. 16,163

8. Naciones Unidas. Convención Marco de las Naciones Unidas Sobre el Cambio Climático. 1992. 5,6

9. Naciones Unidas. Protokolo de Kyoto de la Convención Marco de las Naciones Unidas sobre el cambio climático. 1988. 3,4-13

10. Gobierno Regional Junín. Estrategia Regional de Cambio Climático.2010.6,7,13,28

11. PRAA Perú. Los cambios del clima y sus impactos en la disponibilidad hídrica y principales cultivos en la subcuenca del río Shullcas - Junín. 2012.7

12. PRAA Perú. Cambio Climático en la Cuenca del río Mantaro. Proyecciones para el año 2030. 2012. 30,47

13. Yaranga R, Custodio M. Almacenamiento de Carbono en Pastos Naturales Altoandinos. 2013. Disponible en http://www.sci-agropecu.unitru.edu. pe.

14. Ramírez B; H Ramírez y J Suárez. Captura de carbono y desarrollo radicular de sistemas de uso del suelo en la Amazonia Colombiana. Universidad de la Amazonia, 2010. 9

15. Medrano R, Chupan L, Vila M. Almacenamiento de Carbono en especies predominantes de flora en el lago Chinchaycocha. 2012. Disponible en http:// journals.continental.edu.pe/index.php/apuntes/ article/viewFile/52/51.

16. INIA. Determinación de las Reservas Totales de Carbono en diferentes Sistemas de Uso en el Valle del Mantaro. 2014. 